\title{
Classic progressive supranuclear palsy syndrome
}

INSERM

\section{Source}

INSERM. (1999). Orphanet: an online rare disease and orphan drug data base. Classic progressive supranuclear palsy syndrome. ORPHA:240071

Classical progressive supranuclear palsy, also known as Richardson's syndrome, is the most common clinical variant of progressive supranuclear palsy (PSP; see this term), a rare late-onset neurodeg enerative disease characterized by postural instability, progressive rigidity, supranuclear gaze palsy and mild dementia. 